\title{
PROPAGACIÓN DE PULSOS SOLITÓNICOS EN REDES ÓPTICAS WDM
}

\author{
Claudio Cubillos M. ${ }^{1}$ Ricardo Olivares V. $^{1}$ \\ Recibido el 14 de marzo de 2005, aceptado el 6 de julio de 2005
}

\begin{abstract}
RESUMEN
En el presente trabajo, a través de simulación numérica, se evalúa el desempeño de la propagación de pulsos solitónicos en un sistema de transmisión WDM amplificado mediante EDFAs en cascada. Se modulan 4 portadoras ópticas, con secuencias pseudoaleatorias de 6 bits, a una tasa de $2.5 \mathrm{~Gb} / \mathrm{s}$ cada una. Como una forma de mejorar el desempeño del sistema de transmisión, se ensaya un esquema de fibra con dispersión alternada. Los resultados obtenidos muestran la efectividad de la técnica de administración de la dispersión empleada, reflejada en un menor deterioro de los pulsos propagados.
\end{abstract}

Palabras clave: Solitones ópticos, ecuación no lineal de Schrödinger, método simétrico de paso dividido de Fourier.

\begin{abstract}
The performance of a WDM soliton system provided with a cascade of EDFAs is evaluated by means of computer simulation. Four optical carriers are modulated with a $2.5 \mathrm{~Gb} / \mathrm{s}$ rate, 6-bit-pseudo-random sequence. In order to improve the transmission system performance we introduce a dispersion management scheme by using fibers with dispersion of opposite sign per link. The results obtained show the effectiveness of the dispersion management technique employed, reflected in a smaller distortion of the propagated pulses.
\end{abstract}

Keywords: Optical solitons, non linear Schrödinger equation, symmetrized split step Fourier method.

\section{INTRODUCCIÓN}

El sostenido progreso que ha experimentado la tecnología de las comunicaciones ópticas en el transcurso de la última década ha sido motivado primordialmente por la emergente demanda en servicios de telecomunicaciones (voz, datos, contenidos multimediales, etc.). Los requerimientos por tener anchos de banda cada vez mayores presentan un comportamiento similar a la Ley de Moore, el cual describe el desarrollo en las capacidades de los semiconductores [1]. Dentro de este ámbito, las redes ópticas WDM (Wavelength Division Multiplexing) en conjunto con los amplificadores de fibra dopada con tierras raras (Erbio: $\mathrm{Er}^{3+}$, Neodimio: $\mathrm{Nd}^{3+}, \mathrm{y}$ Praseodimio: $\mathrm{Pr}^{3+}$ ) surgen como la elección natural para satisfacer la creciente demanda de ancho de banda en las estructuras de transporte [2].

Sin embargo, el esquema WDM está sujeto a diversas limitaciones inherentes de la transmisión por fibra óptica, las cuales pueden degradar el desempeño del sistema. Estos efectos incluyen: i) la dispersión cromática de la fibra, que limita la tasa de transmisión debido al ensanchamiento temporal que sufre el pulso transmitido [3]; ii) la no-uniformidad de ganancia del EDFA (Erbium Doped Fiber Amplifier) y el ruido de emisión espontánea amplificado (ASE: Amplified Spontaneous Emission), que degrada la relación señal a ruido (SNR: Signal to Noise Ratio) limitando el rango de longitudes de onda disponibles [4]; iii) diversos efectos no-lineales tales como fenómenos de esparcimiento estimulado inelástico (SRS: Stimulated Raman Scattering, SBS: Stimulated Brillouin Scattering), automodulación de fase (SPM: Self Phase Modulation), modulación de fase cruzada (XPM: Cross Phase Modulation) y mezcla de cuatro ondas (FWM: Four Wave Mixing) que, como resultado de las altas potencias de las señales disponibles en la fibra óptica, distorsionarán las señales transmitidas [5].

En la actualidad, una considerable atención es dirigida al estudio de los solitones ópticos como medio para superar las restricciones e incrementar las tasas de transmisión en la fibra por sobre los $40 \mathrm{~Gb} / \mathrm{s}$ [6]. A pesar de la madurez tecnológica alcanzada por los sistemas solitónicos

\footnotetext{
1 Universidad Técnica Federico Santa María, Departamento de Electrónica, Casilla Postal 110-V, Av. España 1680, Valparaíso-Chile, claudio.cubillos@gmail.com,rov@elo.utfsm.cl
} 
amplificados, estos todavía no han sido desarrollados comercialmente [7].

En el presente trabajo, se evalúa formalmente la propagación multicanal de solitones en sistemas ópticos amplificados, a través de simulaciones en computador. Se emplean modelos numéricos rigurosos para la propagación de pulsos solitónicos WDM en la fibra, a través de cascadas de amplificadores, bajo esquemas con y sin manejo de la dispersión (Dispersion Management).

\section{MODELADO DE LAS COMPONENTES DEL SISTEMA DE TRANSMISIÓN}

El conjunto de transmisión WDM está compuesto por tramos de fibra con dispersión desplazada, cuya atenuación es compensada por una cascada de amplificadores ópticos modelados idealmente [8]. Se admite que el sistema emplea modulación de intensidad con detección directa (IM/DD: Intensity Modulation with Direct Detection).

\section{EI Transmisor}

Las fuentes láser se asumen libres de ruido y de chirp de frecuencia, emitiendo secuencias pseudoaleatorias de 6 bits para cada portadora, en las frecuencias $\mathrm{f}_{1}=194.04$ $\mathrm{THz}\left(\lambda_{1}=1546 \mathrm{~nm}\right), \mathrm{f}_{2}=193.79 \mathrm{THz}\left(\lambda_{2}=1548 \mathrm{~nm}\right)$, $\mathrm{f}_{3}=193.54 \mathrm{THz}\left(\lambda_{3}=1550 \mathrm{~nm}\right), \mathrm{y} \mathrm{f}_{4}=193.29 \mathrm{THz}$ $\left(\lambda_{4}=1552 \mathrm{~nm}\right)$. Cada bit ' 1 ' en la secuencia de datos es simbolizado por un pulso óptico cuya envolvente corresponda a una secante hiperbólica (soliton fundamental). Los pulsos ocupan alrededor de un $20 \%$ del intervalo temporal asignado al bit, para así evitar una posible colisión entre ellos (fenómeno de interacción entre solitones).

\section{Fibras con Dispersión Desplazada}

Los diferentes tipos de fibras ópticas usadas en las diversas distribuciones del enlace son: i) fibras con dispersión desplazada con longitud de onda de cero dispersión en $\lambda_{\mathrm{a}}\left(\mathrm{DSF}_{1}\right.$ : Lucent TrueWave), y ii) fibras con dispersión desplazada con longitud de onda de cero dispersión en $\lambda_{\mathrm{b}}\left(\mathrm{DSF}_{2}\right.$ : Lucent TrueWave-XL). Los parámetros relevantes de cada tipo de fibra son detallados en la Tabla 1.

Un modelo para la propagación de pulsos ópticos en un sistema WDM queda completamente definido a través de un conjunto de ecuaciones no lineales de Schrödinger acopladas. La atenuación, los efectos dispersivos y los fenómenos no-lineales tales como SPM, XPM y SRS, son incorporados apropiadamente en el modelo. Para un sistema WDM de cuatro canales, se obtiene el siguiente conjunto de ecuaciones (donde cada subindice denota el número del canal) [9]:

Tabla 1 Características de los Diferentes Tipos de Fibra.

\begin{tabular}{|c|c|c|c|c|c|}
\hline Fibra & $\begin{array}{c}\alpha \\
(\mathrm{dB} / \mathrm{km})\end{array}$ & $\begin{array}{c}\mathrm{A}_{\text {eff }} \\
\left(\mu \mathrm{m}^{2}\right)\end{array}$ & $\begin{array}{c}\lambda_{\mathrm{zD}} \\
(\mathrm{nm})\end{array}$ & $\begin{array}{c}\mathrm{D}(\text { Banda C) } \\
(\mathrm{ps} /(\mathrm{nm} \mathrm{km}))\end{array}$ & $\begin{array}{c}\text { Pendiente D } \\
\left(\mathrm{ps} /\left(\mathrm{nm}^{2} \mathrm{~km}\right)\right)\end{array}$ \\
\hline $\mathrm{DSF}_{1}$ & 0.20 & 50 & 1529.5 & $0.04 \mathrm{a} 2.59$ & 0.085 \\
\hline $\mathrm{DSF}_{2}$ & 0.20 & 50 & 1566.5 & $-3.10 \mathrm{a}-0.55$ & 0.085 \\
\hline
\end{tabular}

Canal 1:

$$
\begin{aligned}
\frac{\partial A_{1}}{\partial z}+ & \frac{1}{v_{1}} \frac{\partial A_{1}}{\partial T}+\frac{i}{2} \beta_{2}\left(\lambda_{1}\right) \frac{\partial^{2} A_{1}}{\partial T^{2}}+\frac{1}{2} \alpha A_{1} \\
= & i \gamma_{1}\left[\left|A_{1}\right|^{2}+2\left|A_{2}\right|^{2}+2\left|A_{3}\right|^{2}+2\left|A_{4}\right|^{2}\right] A_{1} \\
& +\left[\frac{g_{12}}{2}\left|A_{2}\right|^{2}+\frac{g_{13}}{2}\left|A_{3}\right|^{2}+\frac{g_{14}}{2}\left|A_{4}\right|^{2}\right] A_{1}
\end{aligned}
$$

Canal 2:

$$
\begin{aligned}
\frac{\partial A_{2}}{\partial z}+ & \frac{1}{v_{2}} \frac{\partial A_{2}}{\partial T}+\frac{i}{2} \beta_{2}\left(\lambda_{2}\right) \frac{\partial^{2} A_{2}}{\partial T^{2}}+\frac{1}{2} \alpha A_{2} \\
= & i \gamma_{2}\left[2\left|A_{1}\right|^{2}+\left|A_{2}\right|^{2}+2\left|A_{3}\right|^{2}+2\left|A_{4}\right|^{2}\right] A_{2} \\
& +\left[\frac{g_{21}}{2}\left|A_{1}\right|^{2}+\frac{g_{23}}{2}\left|A_{3}\right|^{2}+\frac{g_{24}}{2}\left|A_{4}\right|^{2}\right] A_{2}
\end{aligned}
$$

Canal 3:

$$
\begin{aligned}
\frac{\partial A_{3}}{\partial z}+ & \frac{1}{v_{3}} \frac{\partial A_{3}}{\partial T}+\frac{i}{2} \beta_{2}\left(\lambda_{3}\right) \frac{\partial^{2} A_{3}}{\partial T^{2}}+\frac{1}{2} \alpha A_{3} \\
= & i \gamma_{3}\left[2\left|A_{1}\right|^{2}+2\left|A_{2}\right|^{2}+\left|A_{3}\right|^{2}+2\left|A_{4}\right|^{2}\right] A_{3} \\
& +\left[\frac{g_{31}}{2}\left|A_{1}\right|^{2}+\frac{g_{32}}{2}\left|A_{2}\right|^{2}+\frac{g_{34}}{2}\left|A_{4}\right|^{2}\right] A_{3}
\end{aligned}
$$

Canal 4:

$$
\begin{aligned}
\frac{\partial A_{4}}{\partial z}+ & \frac{1}{v_{4}} \frac{\partial A_{4}}{\partial T}+\frac{i}{2} \beta_{2}\left(\lambda_{4}\right) \frac{\partial^{2} A_{4}}{\partial T^{2}}+\frac{1}{2} \alpha A_{4} \\
= & i \gamma_{4}\left[2\left|A_{1}\right|^{2}+2\left|A_{2}\right|^{2}+2\left|A_{3}\right|^{2}+\left|A_{4}\right|^{2}\right] A_{4} \\
& +\left[\frac{g_{41}}{2}\left|A_{1}\right|^{2}+\frac{g_{42}}{2}\left|A_{2}\right|^{2}+\frac{g_{43}}{2}\left|A_{3}\right|^{2}\right] A_{4}
\end{aligned}
$$

Usando como referencia al canal $1, \lambda_{1}$ es su longitud de onda; $\mathrm{A}_{1}$ es la amplitud del campo óptico que se propaga; $v_{1}$ es la velocidad de grupo; $\beta_{2}\left(\lambda_{1}\right)$ es la dispersión de la velocidad de grupo la cual viene dada por $-\lambda_{1} D / 2 \pi c ; D$ 
es el parámetro de dispersión; $\alpha$ es la pérdida de la fibra que se asume independiente de la longitud de onda; $\gamma_{1}$ es el coeficiente no-lineal definido como $2 \pi n_{2} \lambda_{1} A_{\text {eff }}$ donde $\mathrm{n}_{2}=3 \times 10^{-20} \mathrm{~m}^{2} / \mathrm{W}$ es el coeficiente de índice no-lineal, y $A_{\text {eff }}$ es el área efectiva del núcleo de la fibra; $\mathrm{g}_{12}, \mathrm{~g}_{13}, \mathrm{~g}_{14}$, son los coeficientes de ganancia Raman entre el canal 1 y los canales 2, 3 y 4, respectivamente. El perfil de ganancia Raman para una fibra de sílice fue aproximado mediante un proceso de interpolación numérica spline cúbica. Para ambas fibras con dispersión desplazada, se utiliza un valor típico de $d D / d \lambda=0.085 \mathrm{ps} /\left(\mathrm{nm}^{2} \mathrm{~km}\right)$ para la pendiente espectral de dispersión. Dada la relevancia de las anteriores ecuaciones, es vital identificar los diferentes factores que inciden sobre ellas. Usando (1) como ejemplo, hay seis términos principales, los cuales representan (partiendo de izquierda a derecha):

1) La evolución de la amplitud del campo óptico con la distancia de propagación en la fibra.

2) La variación de la amplitud del pulso con el tiempo.

3) La dependencia de la dispersión de velocidad de grupo (GVD) en relación a la longitud de onda.

4) La independencia de la atenuación de la fibra respecto de la longitud de onda.

5) La automodulación de fase (SPM) y la modulación de fase cruzada (XPM) del campo óptico.

6) La ganancia Raman (SRS) proporcionada por los canales adyacentes.

El método del paso dividido de Fourier simetrizado (SSSFM: Symmetrized Split-Step Fourier Method) [10] se usa para resolver el sistema de ecuaciones (1)-(4), de manera de simular la propagación de los canales WDM a lo largo de la fibra óptica.

\section{EI Modelo del Amplificador Óptico}

El análisis se realiza a base de un modelo simplificado de un amplificador de fibra dopada con erbio (EDFA: Erbium Doped Fiber Amplifier). El EDFA es tratado como un multiplicador de señal con ganancia de potencia G; el ruido ASE que éste genera es representado a través de una variable aleatoria gaussiana compleja $\tilde{a}_{n}$ que es adicionada a cada componente espectral de la secuencia de pulsos a la salida del amplificador, según la expresión [10]:

$$
\tilde{A}_{\text {out }}(v)=\sqrt{G} \tilde{A}_{\text {in }}(v)+\tilde{a}_{n}(v)
$$

donde $G=\exp \left(\alpha L_{A}\right)$ es la ganancia del amplificador necesaria para compensar las pérdidas de la fibra; $\mathrm{L}_{\mathrm{A}}$ es el espaciamiento entre amplificadores; $\tilde{\mathrm{A}}_{\mathrm{n}}$ y $\tilde{\mathrm{A}}_{\text {out }}$ son las componentes espectrales de la secuencia de pulsos localizados a la entrada y a la salida del amplificador, respectivamente. Las partes real e imaginaria de los componentes espectrales del ruido ASE tienen media nula y varianza igual a la potencia media de ruido blanco, $\mathrm{P}_{\mathrm{ASE}}$, contenida en cada componente del espectro de Fourier [10]:

$$
\sigma^{2}=P_{A S E}=\eta_{s p} \hbar v(G-1) \frac{\Delta v}{2}
$$

donde $\eta_{\mathrm{sp}}$ es el factor de emisión espontánea $\left(\eta_{\mathrm{sp}}=1\right.$ en un amplificador ideal, cuando se obtiene una inversión de población total), $\hbar$ es la constante de Planck, $v$ es la frecuencia óptica, $G$ es la ganancia del amplificador y $\Delta v$ es la ancho de banda ocupada por cada componente de frecuencia. Se asume, además, que la ganancia y la densidad espectral de potencia del ruido ASE en el amplificador son constantes para toda la banda de interés.

\section{RESULTADOS Y DISCUSIONES}

En esta sección se investigaron dos tipos de esquemas de transmisión para un sistema solitónico amplificado WDM. El análisis del desempeño de estos sistemas están basados en la distorsión que experimenta el diagrama de ojo (Eye Diagram) en el receptor, para un canal determinado. Con el propósito de visualizar el diagrama de ojo de manera adecuada, con una mayor nitidez, únicamente se ilustran los dos primeros bits de la trama de datos en el diagrama de ojo correspondiente.

\section{Enlace sin Manejo de Dispersión}

La configuración amplificadora WDM propuesta es ilustrada en la Figura 1, localizada entre un par de WRNs (Wavelength-Routed Nodes). El sistema solitónico WDM está compuesto por cuatro canales, situados entre $1546 \mathrm{y}$ $1552 \mathrm{~nm}(\Delta \lambda=2 \mathrm{~nm})$ con una potencia peak de $1.5 \mathrm{dBm} /$ canal, 15 amplificadores y fibras con dispersión desplazada $\left(\mathrm{DSF}_{1}\right)$. El transmisor láser emite distintas secuencias pseudoaleatorias de 6 bits para cada canal, operando a una tasa de $2.5 \mathrm{~Gb} / \mathrm{s}$. Cada bit ' 1 ' en la secuencia de datos es representado por un solitón fundamental, con un ancho de $\mathrm{T}_{0}=10 \mathrm{ps}$. El ancho y la potencia peak inicial de los pulsos son cuidadosamente escogidos a modo de obtener la condición de solitón fundamental. La trama de datos iniciales para cada portadora es propagada en el primer tramo de fibra de 45 $\mathrm{km}$ de longitud. Las pérdidas consideradas en la fibra $\mathrm{DSF}_{1}$ para la zona espectral de operación es $\alpha=0.2 \mathrm{~dB} / \mathrm{km}$, y los parámetros dispersivos para los respectivos canales son: $\beta_{21}=-1.78 \mathrm{ps}^{2} / \mathrm{km} ; \beta_{22}=-2.00 \mathrm{ps}^{2} / \mathrm{km}, \beta_{23}=-2.22$ $\mathrm{ps}^{2} / \mathrm{km}, \mathrm{y} \beta_{24}=-2.44 \mathrm{ps}^{2} / \mathrm{km}$. 


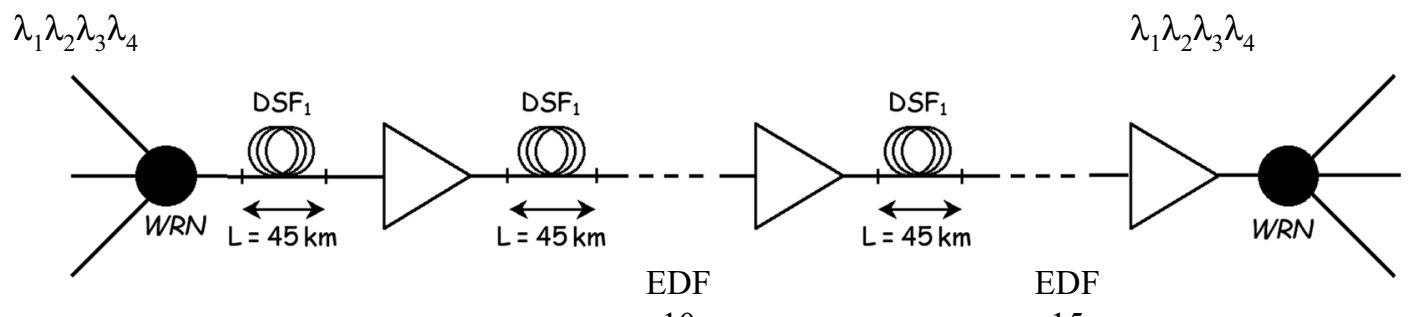

10

15

Fig. 1 Configuración del sistema de transmisión WDM.

Para la configuración amplificadora anteriormente descrita, la ganancia de potencia promedio por amplificador corresponde a $9 \mathrm{~dB}$, a modo de compensar la atenuación en la fibra, y el parámetro $\eta_{\mathrm{sp}}=2$ con objeto de considerar una inversión de población incompleta en el amplificador [11]. Después de propagar en el último tramo de $\mathrm{DSF}_{1}$, la señal óptica recibida es analizada mediante el diagrama de ojo. En la Figura 2 se ilustra el diagrama de ojo para el canal 1 al inicio de la fibra, con el objeto de apreciar el comportamiento preliminar de la trama de datos. Se observa que los pulsos utilizan una leve fracción del intervalo temporal asignado al bit $\left(\mathrm{T}_{\mathrm{B}}=400 \mathrm{ps}\right)$, con la finalidad de minimizar la interacción entre pulsos vecinos.

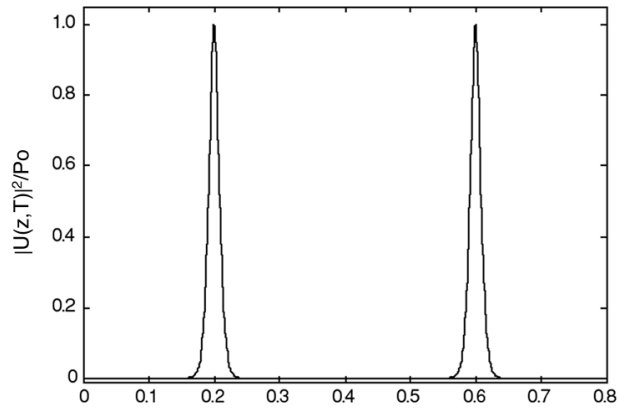

Fig. 2 Diagrama de ojo inicial para $\lambda_{1}=1546 \mathrm{~nm}$.

A continuación, se investiga la influencia de la dispersión, no-linealidades y el ruido ASE sobre la trama de datos para una distancia de $675 \mathrm{~km}$. En la Figura 3 se muestran los respectivos diagramas de ojos para cada canal WDM, calculados a la salida del amplificador \#15. Se puede visualizar que las secuencias de pulsos para cada canal están sujetas a una severa degradación, en relación a los diagramas de ojo calculados al inicio del sistema de transmisión WDM. Este deterioro presente en la trama de datos se visualiza en la forma de un ensanchamiento temporal de los pulsos, más un comportamiento ruidoso oscilatorio en su amplitud. Resulta interesante observar que los canales localizados en el centro del espectro WDM son más afectados en términos de que presentan una mayor degradación del diagrama de ojo, debido fundamentalmente al efecto de la modulación cruzada de fase (XPM) entre los canales adyacentes $\left(\lambda_{1}=1546\right.$ $\left.\mathrm{nm}, \mathrm{y} \lambda_{4}=1552 \mathrm{~nm}\right)$.

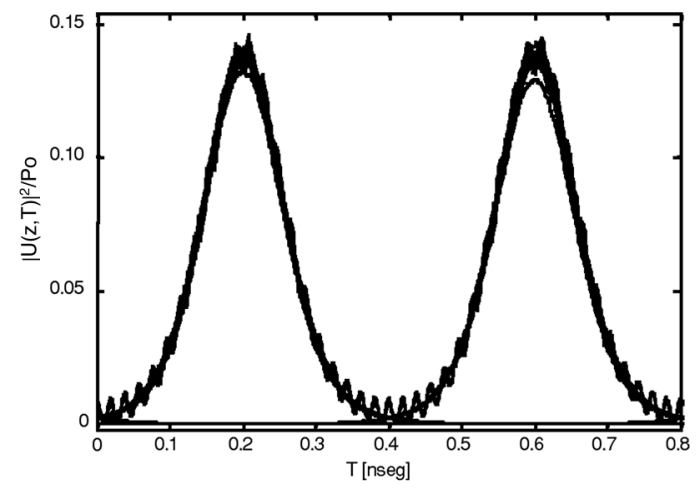

(a)

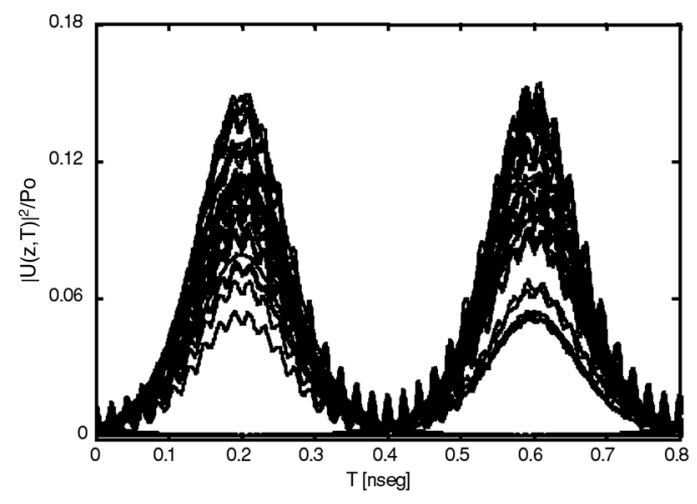

(b)

Fig. 3 Diagrama de ojo de salida para una transmisión de $675 \mathrm{~km}$. Las portadoras ópticas son: (a) $\lambda_{1}=1546 \mathrm{~nm}$. (b) $\lambda_{2}=1548 \mathrm{~nm}$. 


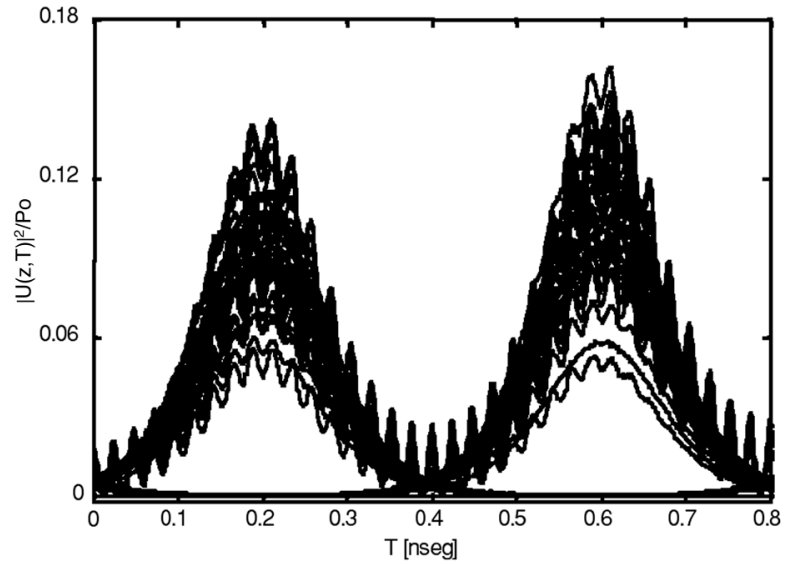

(c)

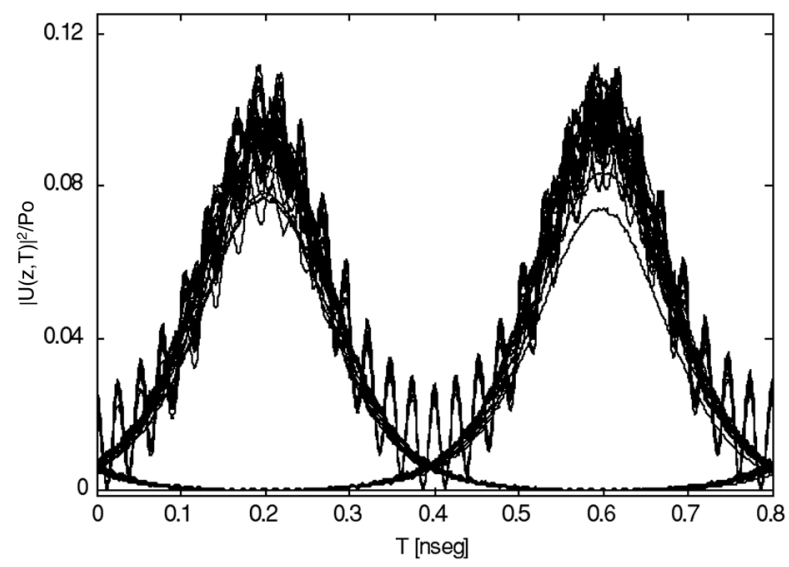

(d)

Fig. 3 Diagramas de ojo de salida para una transmisión de $675 \mathrm{~km}$. Las portadoras ópticas son:

(c) $\lambda_{3}=1550 \mathrm{~nm} ;(\mathrm{d}) \lambda_{4}=1552 \mathrm{~nm}$ (continuación).

En la Figura 4 se aprecia la dispersión total acumulada en función de la distancia de transmisión para el conjunto de los cuatro canales. Mientras que la dispersión acumulada del canal 1 presenta el menor valor al cabo de los $675 \mathrm{~km}$, el canal 4 en cambio exhibe el mayor incremento, debido a las diferencias que manifiestan los valores de la dispersión cromática entre las portadoras WDM. La interacción mutua de la atenuación y la dispersión cromática de la fibra a través del enlace, conllevan inevitablemente al ensanchamiento temporal de los pulsos, ocasionando que estos invadan el intervalo temporal $\mathrm{T}_{\mathrm{B}}$ asignado a los bits vecinos. Este efecto es observado notoriamente para el canal 4, el cual presenta el mayor ensanchamiento temporal después de propagarse en el último tramo de fibra $\mathrm{DSF}_{1}$. Este comportamiento es inaceptable en un sistema de comunicaciones ópticas multicanal, ya que limita la máxima tasa de transmisión como también la distancia de propagación del enlace.

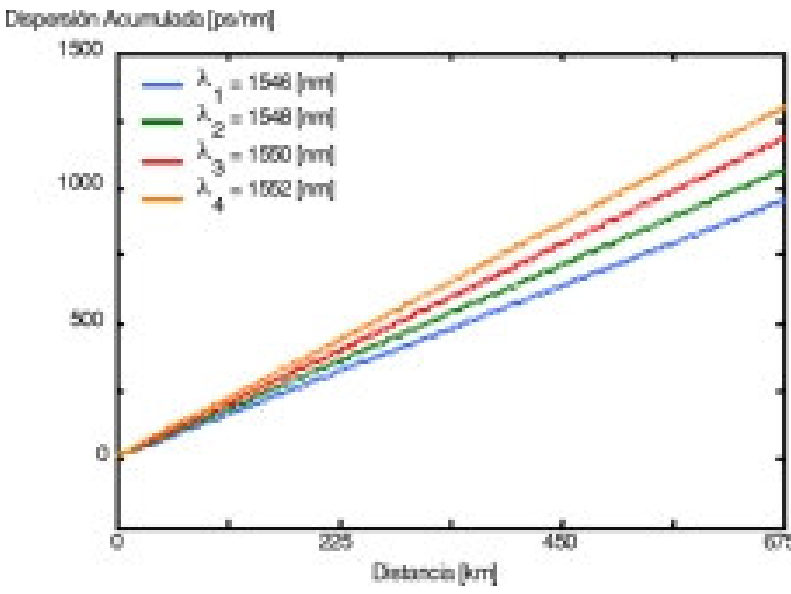

Fig. 4 Dispersión acumulada en función de la distancia para el sistema descrito en la Figura. 1.

\section{Enlace con Administración de la Dispersión}

Como se analizó en la sección precedente, los fenómenos de dispersión cromática y pérdidas en la fibra son los responsables del ensanchamiento temporal de los pulsos solitónicos a medida que se propagan en la fibra óptica. El término administración de la dispersión (Dispersion Management) se refiere a las formas de disminuir o eliminar el ensanchamiento temporal debido a la dispersión cromática, mediante el ajuste de la dispersión promedio del enlace a un valor cercano a cero, usando tramos de fibras con dispersión alternada, cuya dispersión local puede tener un alto valor.

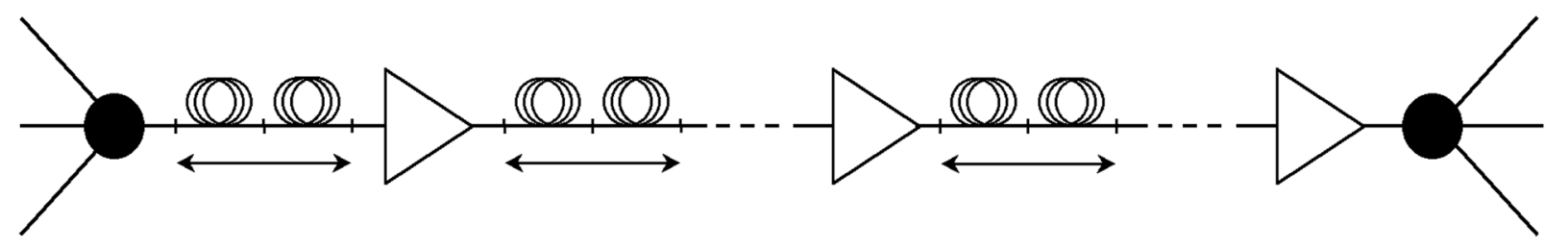

Fig. 5 Configuración del sistema de transmisión WDM, incorporando fibras con dispersión alternada. 
A continuación, se analiza el caso de un esquema con dispersión alternada, en el cual dos fibras con dispersión desplazada, $\mathrm{DSF}_{1}$ y $\mathrm{DSF}_{2}$, son incorporadas al enlace con iguales longitudes $\left(\mathrm{L}_{1}=\mathrm{L}_{2}=22.5 \mathrm{~km}\right)$ en el tramo de fibra entre amplificadores contiguos. La configuración amplificadora propuesta es ilustrada en la figura 5, localizada entre un par de WRNs. El sistema solitónico WDM tiene la misma conformación que en el caso analizado en la Sección A, con la salvedad que en los tramos de fibra entre amplificadores se alternan $\mathrm{DSF}_{1}$ y $\mathrm{DSF}_{2}$. Ambas fibras se asumen con igual atenuación $(\alpha=0.2 \mathrm{~dB} / \mathrm{km})$; los parámetros de dispersión de velocidad de grupo para $\mathrm{DSF}_{1}$ son los mismos indicados en la Sección $\mathrm{A}$, mientras que para $\mathrm{DSF}_{2}$, que estaría operando en el régimen de dispersión normal en la banda $\mathrm{C}$, sus parámetros de dispersión de velocidad de grupo son: $\beta_{21}=2.21 \mathrm{ps}^{2} / \mathrm{km}, \beta_{22}=2.00$ $\mathrm{ps}^{2} / \mathrm{km}, \beta_{23}=1.78 \mathrm{ps}^{2} / \mathrm{km} ; \mathrm{y} \beta_{24}=1.58 \mathrm{ps}^{2} / \mathrm{km}$. En la figura 6 se observan los diagramas de ojo para cada portadora WDM, para una distancia de transmisión de $675 \mathrm{~km}$. Al contrastar estas figuras con el caso sin

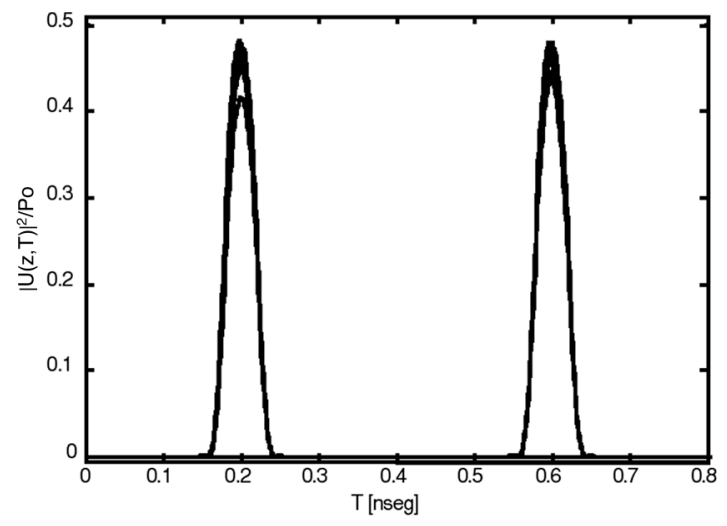

(a)

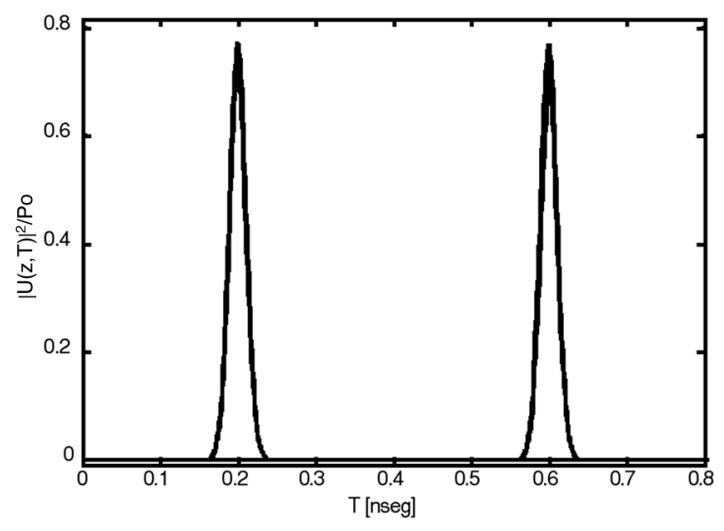

(c) manejo de dispersión (figura 3), se aprecia notoriamente cómo, aplicando la técnica de manejo de la dispersión, es posible minimizar el ensanchamiento temporal, y extender así la distancia de transmisión del enlace. El principio físico está sustentado en que si un pulso se propaga en un mapa de dispersión, su ancho se incrementa en cada sección de fibra con dispersión anómala, pero el pulso se comprime y su ancho es restaurado cuando éste se propaga en secciones de fibra con dispersión normal.

Resulta interesante advertir para la figura 6 (b) que el canal 2 presenta el menor ensanchamiento temporal, además de exhibir una mínima degradación en la intensidad, en relación a los demás canales WDM. Este comportamiento se debe a que la dispersión del canal 2 es seleccionada para ser compensada exactamente por el esquema de fibra con dispersión alternada, como se aprecia en la figura 7. En contraste, la dispersión del canal 1 es sobrecompensada, y la de los canales 3 y 4 es subcompensada, para el esquema de dispersión utilizado.

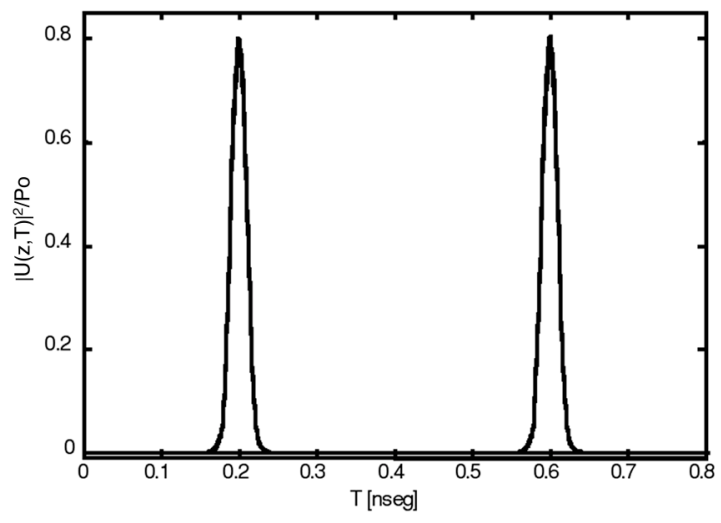

(b)

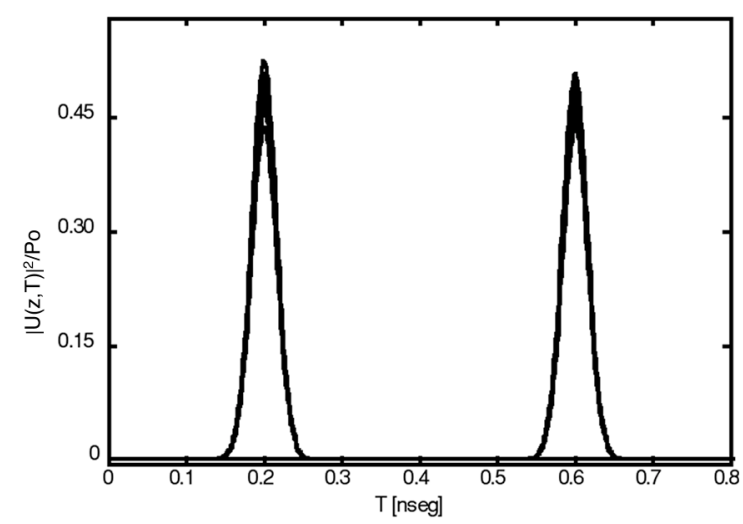

(d)

Fig. 6 Diagramas de ojo de salida para una transmisión de $675 \mathrm{~km}$. Las portadoras ópticos son: (a) $\lambda_{1}=1546 \mathrm{~nm}$; (b) $\lambda_{2}=1548 \mathrm{~nm}$; (c) $\lambda_{3}=1550 \mathrm{~nm}$; (d) $\lambda_{4}=1552 \mathrm{~nm}$. 




Fig. 7 Dispersión acumulada en función de la distancia, alternando (+/-) fibras con dispersión desplazada para el sistema descrito en la figura 5 .

\section{CONCLUSIONES}

En este trabajo se presentó la evaluación de un sistema solitónico amplificado WDM para su aplicación en sistemas de transmisión de largo alcance, basado en simulaciones numéricas rigurosas y bajo condiciones realistas. Las simulaciones indican que el parámetro de dispersión cromática en la fibra óptica juega un rol crucial en el desempeño del sistema solitónico. Asimismo, se confirman las previsiones teóricas de que el empleo de técnicas de control de la dispersión permite un incremento en la distancia de transmisión del enlace. Como desafío futuro se plantea la necesidad de incorporar un modelo más riguroso para el amplificador óptico, de manera que considere el comportamiento real de los EDFAs.

\section{AGRADECIMIENTOS}

La realización de este trabajo fue posible gracias al apoyo parcial de CONICYT (en el contexto del proyecto Fondecyt $\mathrm{N}^{\mathrm{o}} 1010437$ ) y de la UTFSM bajo el proyecto DGIP-230428.

\section{REFERENCIAS}

[1] K. G. Coffman and A. M. Odlyzko, "Internet growth: Is there a "Moore's Law" for data traffic?". AT\&T Labs - Research. http://www.dtc.umn.edu/ $\sim$ odlyzko/doc/internet.moore.pdf
[2] E. Desurvire. "Erbium-Doped Fiber AmplifiersPrinciples and Applications". New York: Wiley Interscience. 1994.

[3] M. J. Potasek, G. P. Agrawal and S. C. Pinault. "Analytic and numerical study of pulse broading in nonlinear dispersive optical fibers". J. Opt. Soc. Amer. B. Vol. 3, No 2, pp. 205-21. 1986.

[4] A. E. Willner and S. M. Hwang. "Transmission of many WDM channels through a cascade of EDFA's in long-distance links and ring networks". J. Lightwave Technol. Vol. 13. Nº5, pp. 802-816. May 1995.

[5] A. Chraplyvy. "Limitations on lightwave communications imposed by optical-fiber nonlinearities". J. Lightwave Technol. Vol. 8, $\mathrm{N}^{\mathrm{o}} 10$, pp. 1548-1557. October 1990.

[6] G. P. Agrawal. "Fiber-Optic Communications Systems". $3^{\text {rd }}$ Ed. John Wiley \& Sons, Inc. New York. 2002.

[7] J. P. Gordon and H. A. Haus. "Random walk of coherently amplified solitons in optical fiber transmission”. Opt. Lett. Vol. 11, pp. 665-667. November 1986.

[8] Lucent Technologies. 2005. http://www.lucent.com

[9] X. Y. Zou, M. I. Hayee, S. M. Hwang and A. E. Willner. "Limitations in $10 \mathrm{~Gb} / \mathrm{s}$ WDM optical-fiber transmission when using a variety of fiber types to manage dispersion and nonlinearities". J. Lightwave Technol. Vol. 14, Nº, pp. 1144-1152. June 1996.

[10] C. Cubillos. "Propagación de Pulsos Solitónicos en Sistemas Ópticos Amplificados". Tesis para optar al título de Ingeniero Civil Electrónico, Universidad Técnica Federico Santa María, Valparaíso, Chile. Agosto 2004.

[11] D. Marcuse. "Single-channel operation in very long nonlinear fibers with optical amplifiers at zero dispersion". J. Lightwave Technol. Vol. 9, № 3, pp. 356-361. March 1991. 